\title{
On the Time-Delay of Simple Scattering Systems
}

\author{
Ph. A. Martin
}

Laboratoire de Physique Théorique, Ecole Polytechnique Fédérale, CH-1006 Lausanne, Switzerland

\begin{abstract}
A new rigorous and simple study of the time-delay formula is presented.
\end{abstract}

\section{Introduction}

The time-delay of a scattering process may intuitively be considered to be the difference between the time spent by the colliding particles within the region of mutual interaction and the time that they would have spent in the same region had they moved freely. Consider a simple scattering system $\left(H, H_{0}\right)$ on a Hilbert space $\mathscr{H}$ with free evolution $U_{t}=\exp \left(-i H_{0} t\right)$ and total evolution $V_{t}=\exp (-i H t)$ [1]. The wave operators $\Omega_{ \pm}=s-\lim _{t \rightarrow \pm \infty} \exp (i H t) \exp \left(-i H_{0} t\right)$ are assumed to be complete and the scattering operator $S=\Omega_{+}^{*} \Omega_{-}$is unitary. Let $P_{r}$ be the projection operator on a region $\Sigma_{r}$ in configuration space in which the distance between particles does not exceed $r$. If $\psi_{t}=V_{t} \Omega_{-} \varphi$ is a scattering state which behaves as the freely evolving state $\varphi_{t}=U_{t} \varphi$ as $t \rightarrow-\infty$, the mean times spent in $\Sigma_{r}$ by the interacting and the free particles are respectively:

$$
\int_{-\infty}^{\infty}\left(\psi_{t}, P_{r} \psi_{t}\right) d t \text { and } \int_{-\infty}^{\infty}\left(\varphi_{t}, P_{r} \varphi_{t}\right) d t
$$

The time-delay for the scattering state $\Omega_{-} \varphi$ and the region $\Sigma_{r}$ is then defined by $[2,3]$ :

$$
T_{r}(\varphi)=\int_{-\infty}^{\infty}\left[\left(\psi_{t}, P_{r} \psi_{t}\right)-\left(\varphi_{t}, P_{r} \varphi_{t}\right)\right] d t
$$

This definition raises three mathematical questions which are in logical order:

(i) Is the expression (1) meaningful for finite $r$ and suitable states $\varphi$ ?

(ii) Does there exist a time-delay $T(\varphi)=\lim _{r \rightarrow \infty} T_{r}(\varphi)$ for infinite space region?

(iii) Is $T(\varphi)$ related to the classical formula of Eisenbud and Wigner [4] asserting that the time-delay is expressed by the derivative of the phase shift with respect to energy? 
Question (i) can clearly be affirmatively answered on the grounds that scattering states propagate away from any bounded space region so that time integrals occuring in (1) are finite.

The point (ii), which is likely to be true for sufficiently short ranged interactions, is mathematically more delicate and it has received two different treatments using time dependent methods. The first one [3] shows the existence of the limit with the help of an abstract analysis based on the condition that the difference of the resolvants $(H-z)^{-1}-\left(H_{0}-z\right)^{-1}$ belongs to the trace-class of operators. The second one [5] makes use of the concept of $H$-smoothness and of commutator techniques developed in potential scattering. Finally in [3] the connection (iii) between time-delay and phase shift (which is not studied in [5]) is obtained by the rather elaborated tools of the theory of the spectral displacement function due to Birman and Krein (see ref. in [3]).

The purpose of this note is to present a rigorous and elementary derivation of the Eisenbud-Wigner formula, which is independent of the previously quoted works. Our study of time-delay relies essentially on simple asymptotic properties in time of scattering systems. While being of slightly less general applicability than that of ref. [3], it uses much more direct and transparent arguments and thus it has the merit of simplicity and conciseness.

\section{The Time-Delay Formula}

The main element of our demonstration consists in the following proposition which we now state and prove.

Proposition 1. Let $U_{t}$ be a strongly continuous unitary group on a Hilbert space $\mathscr{H}$ whose generator $H_{0}=\int \lambda d E_{\lambda}$ has an absolutely continuous spectral family $E_{\lambda}$. Let $P_{r}$ be a sequence of projections on $\mathscr{H}$ converging strongly to $I$ as $r \rightarrow \infty$, and $A=\int A(\lambda) d E_{\lambda}$ be an operator function of $H_{0}$ (in the sense of functional calculus). Let $\varphi$ and $\chi$ be in $\mathscr{H}$. We assume:

(i) the functions $t \rightarrow\left\|P_{r} U_{t} \varphi\right\|$ and $t \rightarrow\left\|P_{r} U_{t} \chi\right\|$ belong to $\mathscr{L}^{1}(R, d t)$ for each $r$.

(ii) $A(\lambda)$ is differentiable and $A(\lambda)$ together with its derivative are Fourier transforms of $\mathscr{L}^{1}$ functions. Then

$$
\lim _{r \rightarrow \infty} \int_{0}^{\infty}\left(U_{t} \varphi,\left[P_{r}, A\right] U_{t} \chi\right) d t=-i\left(\varphi, \frac{d A}{d H_{0}} \chi\right) .
$$

From the assumption (ii), $A$ and $\frac{d A}{d H_{0}}=\int \frac{d A(\lambda)}{d \lambda} d E_{\lambda}$ are bounded operators on $\mathscr{H}$. Notice that in view of (i) the time integral in (2) converges absolutely.

Proof. Set $\varphi_{t}=U_{t} \varphi$ and $\chi_{t}=U_{t} \chi$. By the very definition of $A$ and $U_{t}$ one has:

$$
\begin{aligned}
(\varphi, A \chi) & =\int A(\lambda) d\left(\varphi, E_{\lambda} \chi\right)=\int d\left(\varphi, E_{\lambda} \chi\right) \int \tilde{A}(\alpha) e^{i \alpha \lambda} d \alpha \\
& =\int d \alpha \tilde{A}(\alpha) \int e^{i \lambda \alpha} d\left(\varphi, E_{\lambda} \chi\right)=\int d \alpha \tilde{A}(\alpha)\left(\varphi_{\alpha}, \chi\right) .
\end{aligned}
$$

Since the Fourier transform $\tilde{A}(\alpha)$ of $A(\lambda)$ belongs to $\mathscr{L}^{1}$ the exchange of integrals is allowed by Fubini's theorem. 
Inserting (3) in (2) we get:

$$
\begin{aligned}
\int_{0}^{\infty}\left(\varphi_{t},\left[P_{r}, A\right] \chi_{t}\right) d t & =\int_{0}^{\infty} d t \int d \alpha \tilde{A}(\alpha)\left[\left(\varphi_{t}, P_{r} \chi_{t-\alpha}\right)-\left(\varphi_{t+\alpha}, P_{r} \chi_{t}\right)\right] \\
& =\int d \alpha \tilde{A}(\alpha) \int_{0}^{\infty} d t\left[\left(\varphi_{t}, P_{r} \chi_{t-\alpha}\right)-\left(\varphi_{t+\alpha}, P_{r} \chi_{t}\right)\right] \\
& =\int d \alpha \tilde{A}(\alpha) \int_{0}^{\alpha}\left(\varphi_{t}, P_{r} \chi_{t-\alpha}\right) d t
\end{aligned}
$$

Owing to the fact that the integrand is majorized by the $\mathscr{L}^{1}$ function $|\tilde{A}(\alpha)|\left(\left\|P_{r} \varphi_{t}\right\|\|\chi\|+\left\|P_{r} \chi_{t}\right\|\|\varphi\|\right)$ in $\alpha$ and $t$, the exchange of the $\alpha$ and $t$ integrals is also possible.

(4) results of the change of variable $t+\alpha \rightarrow t$ in the second time integral. The integrand of (4) is majorized uniformly in $r$ by an integrable function of $\alpha$

$$
\left|\tilde{A}(\alpha) \int_{0}^{\alpha}\left(\varphi_{t}, P_{r} \chi_{t-\alpha}\right) d t\right| \leqq\left|\tilde{A}(\alpha) \int_{0}^{\alpha}\|\varphi\|\|\chi\| d t\right|=\|\varphi\|\|\chi\||\alpha \tilde{A}(\alpha)| .
$$

Therefore the dominated convergence theorem applies in (4) yielding

$$
\lim _{r \rightarrow \infty} \int_{0}^{\infty}\left(\varphi_{t},\left[P_{r}, A\right] \chi_{t}\right) d t=\int d \alpha \tilde{A}(\alpha) \int_{0}^{\alpha} \lim _{r \rightarrow \infty}\left(\varphi_{t}, P_{r} \chi_{t-\alpha}\right) d t=\int d \alpha \tilde{A}(\alpha) \alpha\left(\varphi_{\alpha}, \chi\right)
$$

The same argument which gave (3) now shows that this last expression precisely equals $-i\left(\varphi, \frac{d A}{d H_{0}} \chi\right)$ and this concludes the proof.

Consider a scattering system $\left(H, H_{0}\right)$ whose free Hamiltonian $H_{0}$ is assumed to have an absolutely continuous simple spectrum on $\mathscr{H}$. Then the scattering operator, which commutes with $H_{0}$, is necessarily given by a function $S(\lambda)$ on the spectrum of $H_{0}$. If such a scattering system has suitable asymptotics in time (i.e. the asymptotic condition holds with a sufficiently fast rate of convergence), Proposition 1 can be used for a straightforward derivation of the EisenbudWigner formula. This is the content of Proposition 2.

Proposition 2. Let $\mathscr{D}$ be the set of Schwartz functions with compact support on the spectrum of $H_{0}$ and let $\varphi \in \mathscr{D}$.

a) Let $P_{r}$ be a sequence of projections on $\mathscr{H}$ converging strongly to $I$ as $r \rightarrow \infty$, and such that the functions $t \rightarrow\left\|P_{r} U_{t} \varphi\right\|$ and $t \rightarrow\left\|P_{r} U_{t} S \varphi\right\|$ belong to $\mathscr{L}^{1}(R, d t)$ for each $r$.

b) Assume that the functions $t \rightarrow\left\|\left(V_{t} \Omega_{-}-U_{t}\right) \varphi\right\|$ and $t \rightarrow\left\|\left(V_{t} \Omega_{-}-U_{t} S\right) \varphi\right\|$ are integrable at $t \rightarrow-\infty$ and $t \rightarrow+\infty$ respectively.

c) Suppose moreover that $S(\lambda)$ is twice continuously differentiable.

Then $\lim _{r \rightarrow \infty} T_{r}(\varphi)=-i\left(\varphi, S^{*} \frac{d S}{d H_{0}} \varphi\right)$.

Proof. Set $\psi_{t}=V_{t} \Omega_{-} \varphi$ and $\varphi_{t}=U_{t} \varphi,\|\varphi\|=1$. We evaluate separately in (1) the integral over negative and positive times. For negative times the integrand is 
majorized uniformly in $r$ by an integrable function at $t \rightarrow-\infty$ :

$$
\left|\left(\psi_{t}, P_{r} \psi_{t}\right)-\left(\varphi_{t}, P_{r} \varphi_{t}\right)\right| \leqq\left|\left(\left(\psi_{t}-\varphi_{t}\right), P_{r} \varphi_{t}\right)\right|+\left|\left(\psi_{t}, P_{r}\left(\psi_{t}-\varphi_{t}\right)\right)\right| \leqq 2\left\|\psi_{t}-\varphi_{t}\right\| .
$$

We have similarly for positive times

$$
\left|\left(\psi_{t}, P_{r} \psi_{t}\right)-\left(S \varphi_{t}, P_{r} S \varphi_{t}\right)\right| \leqq 2\left\|\psi_{t}-S \varphi_{t}\right\|
$$

which is integrable at $t \rightarrow+\infty$.

Hence by dominated convergence the integral over the negative times does not contribute to the time-delay in the limit $r \rightarrow \infty$ and we are left with

$$
\begin{aligned}
\lim _{r \rightarrow \infty} T_{r}(\varphi) & =\lim _{r \rightarrow \infty} \int_{0}^{\infty}\left[\left(S \varphi_{t}, P_{r} S \varphi_{t}\right)-\left(\varphi_{t}, P_{r} \varphi_{t}\right)\right] d t \\
& =\lim _{r \rightarrow \infty} \int_{0}^{\infty}\left(S \varphi_{t},\left[P_{r}, S\right] \varphi_{t}\right) d t .
\end{aligned}
$$

In obtaining (5), use has been made of the unitarity of the $S$ operator. We now apply Proposition 1 to (5) in the following way. Let $g(\lambda)$ be a function on the spectrum of $H_{0}$ which belongs to $\mathscr{D}$ and equals one on the support of $\varphi$. Then $S_{g} \varphi=S \varphi$ with $S_{g}(\lambda)=g(\lambda) S(\lambda)$, and $S_{g}$ can be substituted to $S$ in (5). With the help of two integrations by part one verifies immediately from c) that $S_{g}(\lambda)$ as well as its first derivative are Fourier transforms of $\mathscr{L}^{1}$ functions. Therefore Proposition 1 applies to (5) with $S_{g}$ in place of $S$, and we get

$$
\lim _{r \rightarrow \infty} T_{r}(\varphi)=-i\left(S_{g} \varphi, \frac{d S_{g}}{d H_{0}} \varphi\right)=-i\left(\varphi, S^{*} \frac{d S}{d H_{0}} \varphi\right) .
$$

Proposition 2 gives in a simple manner positive answers to the questions (ii) and (iii) stated in the introduction. It remains to exhibit a class of interactions for which conditions b) and c) hold.

\section{Potential Scattering}

Let $H_{0}=-\Delta$ be the Laplacian acting on $\mathscr{L}^{2}\left(R^{3}\right)$ and $H=-\Delta+\mathscr{V}(x), x=|\boldsymbol{x}|$. $\mathscr{V}(x)$ is a spherically symmetric potential for which the asymptotic condition holds with complete wave operators. (See [6] X §3.)

Lemmas 1 and 2 supplement the asymptotic condition with an information on the rate of convergence as $t$ goes to infinity.

Lemma 1. Assume that $\left(1+|\boldsymbol{x}|^{1+\varepsilon}\right) \mathscr{V}(\boldsymbol{x}) \in \mathscr{L}^{2}\left(R^{3}\right)$ for some $\varepsilon>0$. Let $\varphi$ be a vector whose momentum representation $\tilde{\varphi}(\boldsymbol{k})$ is a Schwartz $\mathscr{D}$ function with no support at the origin $\boldsymbol{k}=0$. Then $t \rightarrow\left\|\left(V_{t} \Omega_{-}-U_{t}\right) \varphi\right\|$ is integrable at $t \rightarrow-\infty$.

Proof. A standard estimate yields for $\varphi \in \mathscr{D}$

$$
\left\|\left(V_{t} \Omega_{-}-U_{t}\right) \varphi\right\| \leqq \int_{-\infty}^{t}\left\|\mathscr{V} U_{\tau} \varphi\right\| d \tau
$$


The norm $\left\|\mathscr{V} U_{t} \varphi\right\|$ is evaluated with the help of the explicit form of the kernel of the free propagator in configuration space, noting that $\left(\frac{1}{2 \pi}\right)^{3 / 2} \int \varphi(\boldsymbol{y}) d^{3} y=\tilde{\varphi}(0)=0$,

$$
\begin{aligned}
& \left\|\mathscr{V} U_{t} \varphi\right\|^{2}=\left(\frac{1}{4 \pi|t|}\right)^{3} \int d^{3} x|\mathscr{V}(\boldsymbol{x})|^{2}\left|\int d^{3} y\left(\exp \left(\frac{i|\boldsymbol{x}-\boldsymbol{y}|^{2}}{4 t}\right)-1\right) \varphi(\boldsymbol{y})\right|^{2} \\
& =\left(\frac{1}{4 \pi|t|}\right)^{3}\left(\frac{1}{4|t|}\right)^{2 \mu} \int d^{3} x|\mathscr{V}(\boldsymbol{x})|^{2}\left|\int d^{3} y\left[\frac{\exp \left(\frac{i|\boldsymbol{x}-\boldsymbol{y}|^{2}}{4 t}\right)-1}{\left(\frac{|\boldsymbol{x}-\boldsymbol{y}|^{2}}{4 t}\right)^{\mu}}\right]\right| \boldsymbol{x}-\left.\left.\boldsymbol{y}\right|^{2 \mu} \varphi(\boldsymbol{y})\right|^{2} .
\end{aligned}
$$

Taking into account that $\left|\frac{e^{i x}-1}{x^{\mu}}\right| \leqq 2,|x-y|^{2 \mu} \leqq 2\left(|x|^{2 \mu}+|\boldsymbol{y}|^{2 \mu}\right)$ for $0 \leqq \mu \leqq 1$, this integral is estimated as follows:

$$
\left\|\mathscr{V} U_{t} \varphi\right\|^{2} \leqq \frac{C_{1}}{|t|^{3+2 \mu}} \int d^{3} x|\mathscr{V}(\boldsymbol{x})|^{2}\left(\int d^{3} y\left(|\boldsymbol{x}|^{2 \mu}+|\boldsymbol{y}|^{2 \mu}\right)|\varphi(\boldsymbol{y})|\right)^{2}
$$

$\int|\varphi(y)| d^{3} y$ and $\int|\boldsymbol{y}|^{2 \mu}|\varphi(y)| d^{3} y$ are clearly finite with our choice of $\varphi$ and hence

$$
\left\|\mathscr{V} U_{t} \varphi\right\| \leqq \frac{C_{2}}{|t|^{3 / 2+\mu}}\left\|\left(1+|x|^{2 \mu}\right) \mathscr{V}\right\|_{2} .
$$

Choosing $\frac{1}{2}<\mu \leqq \frac{1}{2}(1+\varepsilon)$ and integrating the inequality (6) with respect to $t$ yields the desired result.

Lemma 2. Let $\mathscr{V}(x)$ be as in Lemma 1. Consider the decomposition $\oplus \sum_{\substack{l=0 \\|m| \leqq l}}^{\infty} \mathscr{H}_{l m}$ of $\mathscr{L}^{2}\left(R^{3}\right)$ into the direct sum of invariant subspaces under the rotation group. Let $\varphi \in \mathscr{H}_{l m}$ be a vector whose representative $\varphi_{l m}(k), k=|\boldsymbol{k}|$, is a $\mathscr{D}$ function with no support at the origin. If $S_{l}(k)$ is three times continuously differentiable, then $t \rightarrow$ $\left\|\left(V_{t} \Omega_{-}-U_{t} S\right) \varphi\right\|$ is integrable at $t \rightarrow+\infty$.

Proof. From the fact that the wave operators are complete we get

$$
\left\|\left(V_{t} \Omega_{-}-U_{t} S\right) \varphi\right\|=\left\|\left(V_{t} \Omega_{+}-U_{t}\right) S \varphi\right\| \leqq \int_{t}^{\infty}\left\|\mathscr{V} U_{\tau} S \varphi\right\| d \tau .
$$

Thus the argument leading to (6) can be reproduced without modification and the conclusion of the Lemma will hold, provided that $\int d^{3} y|(S \varphi)(y)|$ and $\int d^{3} y|y|^{2 \mu}|(S \varphi)(y)|$ are finite for some $\mu>\frac{1}{2}$. In view of the asymptotic properties of the spherical Bessel function $j_{l}$ we conclude from three successive integrations by part that $(S \varphi)(y)=4 \pi Y_{l m}(\vartheta, \gamma) \int k^{2} d k_{l}(k y) S_{l}(k) \varphi_{l m}(k), y=(y, \vartheta, \gamma)$, can be written in the form $Y_{l m}(\vartheta, \gamma) \frac{f(y)}{1+y^{4}}$ with $f(y) \in \mathscr{L}^{2}\left(R^{+}, d y\right)$.

Hence by Schwartz inequality we have

$$
\int d^{3} y|y|^{2 \mu}|(S \varphi)(y)| \leqq C\|f\|_{2}\left(\int_{0}^{\infty}\left(\frac{y^{2(1+\mu)}}{1+y^{4}}\right)^{2} d y\right)^{1 / 2}
$$

which is finite for $\mu<\frac{3}{4}$. 
Finally Lemma 3 links the differentiability of the $S$ matrix to the decrease of the potential at infinity. We merely sketch its proof which uses standard methods [7].

Lemma 3. Assume that $\int_{0} x^{p}|\mathscr{V}(x)| d x<\infty . p=0,1 \ldots s, s$ being a positive integer. Then $S_{l}(k)$ is continuously differentiable for $k \neq 0$ up to order $s$.

Proof. From the wellknown relation between the Jost solution $f(k, x)$ of the radial Schrödinger equation and the scattering matrix [7, Chap. 5] it is sufficient to show that $f(k, x)$ together with $\frac{\partial}{\partial x} f(k, x)$ are $s$ time continuously differentiable in $k$ for some $x>0 . f(k, x)=\sum_{n=0}^{\infty} f_{n}(k, x)$, where the iterated functions $f_{n}(k, x)$ obey the equation

$$
f_{n}(k, x)=\int_{x}^{\infty} B(k, x, y) \mathscr{V}(y) f_{n-1}(k, y) d y
$$

with kernel $B(k, x, y)=\frac{i}{2 k}\left(f_{0}(k, y) f_{0}(-k, x)-f_{0}(k, x) f_{0}(-k, y)\right), \quad f_{0}(k, x)=$ $(-i)^{l+1} k x h_{l}^{(2)}(k x), h^{(2)}$ being the second spherical Hankel function. When $k$ and $x$ lie in the domains $k \geqq k_{0}>0, x \geqq 1$, we can find constants $C_{1}$ and $C_{2}$ independent of $k$ and $x$ such that

$$
\left|\frac{\partial^{p}}{\partial k^{p}} f_{0}(k, x)\right| \leqq C_{1} x^{p},\left|\frac{\partial^{p}}{\partial k^{p}} B(k, x, y)\right| \leqq C_{2} x^{p} y^{p}, \quad p=0,1, \ldots, s .
$$

Then one proves easily by induction that $f_{n}(k, x)$ is continuously differentiable up to order $s$ and that for $k \geqq k_{0}$ and $x \geqq 1$,

$$
\left|\frac{\partial^{p}}{\partial k^{p}} f_{n}(k, x)\right| \leqq C_{1}(n+1)^{p} \frac{x^{p}}{n !}\left(C_{2} \int_{x}^{\infty} y^{p}|\mathscr{V}(y)| d y\right)^{n} \quad \begin{aligned}
& p=0,1, \ldots, s \\
& n=0,1,2, \ldots
\end{aligned}
$$

Thus the series of derivatives converges uniformly for $k \geqq k_{0}$, from which the differentiability properties of $f(k, x)$ follow.

Let $\mathscr{V}(x)$ be a locally square integrable spherically symmetric potential which is $O\left(\frac{1}{x^{\gamma}}\right), x \rightarrow \infty$, with $\gamma>4$ and consider the restriction of the scattering system $(-\Delta,-\Delta+\mathscr{V}(x))$ to a subspace $\mathscr{H}_{l m}$. Then, on $\mathscr{H}_{l m}, H_{0}$ has a simple spectrum and one concludes from Lemmas 1, 2, and 3 that Proposition 2 applies for all $\varphi \in \mathscr{H}_{l m}$ which are $\mathscr{D}$ functions on the energy spectrum having no support at the origin $\lambda=|\boldsymbol{k}|^{2}=0$. Notice that condition a) of Proposition 2 holds for projections $P_{r}$ associated with any family of bounded space regions $\Sigma_{r}$ approaching $R^{3}$ as $r \rightarrow \infty$. Therefore the time-delay for infinite space region is independent of its finite space-region approximating sequences.

Our result should be compared with that of [3] where it is shown that the energy shell components $T_{r}(\lambda)$ of the time-delay converge in the distribution sense to the derivative of the phase shift, when the difference of the resolvants 
belongs to the trace-class. Since the latter condition is fulfilled in potential scattering if and only if $\mathscr{V}(x)=O\left(\frac{1}{x^{\gamma}}\right), \gamma>3$ [8], it allows a slightly slower decrease of the potential at infinity than that we have to require for the validity of the present analysis.

\section{References}

1. Jauch, J. M.: Helv. Phys. Acta 31, 127 (1958)

2. Jauch, J. M., Marchand, J. P.: Helv. Phys. Acta 40, 217 (1968)

3. Jauch, J. M., Sinha, K., Misra, B.: Helv. Phys. Acta 45, 398 (1972)

4. Wigner, E. P.: Phys. Rev. 98, 145 (1955)

5. Lavine, R.: In: Scattering in Mathematical Physics, p. 141. Dordrecht: D. Reidel Publishing Company 1974

6. Kato,T.: Perturbation Theory for Linear Operator. Berlin-Heidelberg-New York: Springer 1966

7. Alfaro, de, V., Regge, T.: Potential Scattering. Amsterdam: North-Holland 1965

8. Martin, Ph., Misra, B.: J. Math. Phys. 14, 997 (1973)

Communicated by W. Hunziker

Received August 20, 1975 
